\title{
Relation Between Nutritional Risk Factors and Prevalence of Coronary Artery Disease in Smokers and Non-Smokers Resident in Jeddah Governorate, Saudi Arabia
}

\author{
Mostafa Osfor ${ }^{1, ~ *}$, Ahmed Mohamed Ashshi ${ }^{2}$, Mohamed Abo Bakr Baslama ${ }^{2}$, \\ Ammar Abdallah Attar ${ }^{2,3,4}$, Samaa El-Soadaa', Mohamed Mohamed Khereldeen ${ }^{2}$ \\ ${ }^{1}$ Department of Clinical Nutrition, Faculty of Applied Medical Sciences, Umm Al-Qura University, Makkah, Saudi Arabia \\ ${ }^{2}$ Department of Laboratory Medicines, Faculty of Applied Medical Sciences, Umm Al-Qura University, Makkah, Saudi Arabia \\ ${ }^{3}$ Science \& Technology Unit, General Presidency for the Holy Mosque \& Prophet Holy Mosque Affairs, Makkah, Saudi Arabia \\ ${ }^{4}$ Innovation Department, Associate Executive Department of Innovation \& Corporate Integration, King Abdullah Medical City in Holy Capital, \\ Makkah, Saudi Arabia
}

Email address:

mosfor@yahoo.co.uk (M. Osfor)

\section{To cite this article:}

Mostafa Osfor, Ahmed Mohamed Ashshi, Mohamed Abo Bakr Baslama, Ammar Abdallah Attar, Samaa El-Soadaa, Mohamed Mohamed Khereldeen. Relation Between Nutritional Risk Factors and Prevalence of Coronary Artery Disease in Smokers and Non-Smokers Resident in Jeddah Governorate, Saudi Arabia. Science Journal of Public Health. Vol. 3, No. 3, 2014, pp. 375-383. doi: 10.11648/j.sjph.20150303.22

\begin{abstract}
National guidelines for coronary artery disease (CAD) risk reduction have focused on high-risk families, yet little is known about prevalence of risk factors in general population. To determine the magnitude of the problem relative to the general population, a community-based pilot study of the widely accepted CAD risk factors was carried out over the period of 12 months on a random sample of apparently healthy adults $(\mathrm{n}=880)$, aged $18-60$ years, living in Jeddah Governorate. Three hundred eighty eight (388) subjects (156 males and 232 females) residing in Jeddah town (urban group) and four hundred ninety two (492) subjects (264 males and 228 females) adjoining different rural centers in Jeddah Governorate (rural group) participated. The study was based on complete history taking, prevalence of family history of CAD, obesity, hypertension, diabetes mellitus, and history of current cigarettes smoking. Body Mass Index (BMI) was significantly high in urban than rural men and in urban than rural women. Systolic hypertension was recorded in $30.8 \%$ of urban men, $25.8 \%$ of rural men, $24.6 \%$ of rural women and $13.8 \%$ of urban women. Total caloric intake per day was much increased in urban than rural women and in urban than rural men. Serum total cholesterol was significantly higher in urban men and women than in rural men and women respectively. Total cholesterol / HDL ratio showed insignificant difference in the studied groups. Serum $\mathrm{Cu}^{+2} \mathrm{and} \mathrm{Mg}^{+2}$ were significantly higher in urban than rural women. Serum $\mathrm{Zn}^{+2}$ and $\mathrm{Zn}^{+2} / \mathrm{Cu}^{+2}$ ratios showed insignificant difference between the studied groups. Meanwhile, rural smokers showed significant increase in serum level of total cholesterol and $\mathrm{Cu}^{+2}$ with low $\mathrm{Zn}^{+2}$ levels. BMI correlated significantly with serum $\mathrm{Mg}^{+2}$ in urban women and serum $\mathrm{Cu}^{+2}$ in urban men. The prevalence risk factors for $\mathrm{CAD}$ were markedly raised in rural women and urban men, while rural men showed high prevalence of absence of risk factors. Prevalence of major risk factors increases in rural areas and may be due to dietary and life style changes. Serum trace elements like $\mathrm{Cu}^{+2}, \mathrm{Zn}^{+2}$ and $\mathrm{Mg}^{+2}$ may predict coronary ischemia as they correlated significantly with BMI and total caloric daily intake which might be affected by current smoking. Further studies of CAD risk factors, their predictive capacity, heritability estimates, and the degree of which they are amenable to treatment are actually needed.
\end{abstract}

Keywords: Nutritional Habitats, Smoking, Coronary Artery Diseases

\section{Introduction}

The prevention of coronary artery disease (CAD) is based on the control of several factors with this disease and is suspected to play a pathogenic role, defined as "risk factors".
Most of the epidemiological studies that have evaluated coronary risk factors have measured either mortality or morbidity of CAD. ${ }^{[1]}$ Delineating the role of the diet in CAD 
in adults is important for guiding dietary recommendation and prevention of CAD. Dietary factors such as total caloric intake, saturated fats, polyunsaturated fatty acids, trace elements, certain proteins and fibers have all been suggested as playing a role in the pathogenesis of CAD. ${ }^{[2]}$ Total fat intake is not strongly associated with $\mathrm{CAD}$, but may contribute to obesity. Association between total fat intake and CAD are primarily mediated through the saturated fatty acids components. [3] Obesity, especially truncal type (android obesity) had statistically significant detrimental effects on lipid profile, being associated with higher total and low density lipoprotein cholesterol (LDL-ch), and high density lipoprotein cholesterol (HDL-ch). [4],[5] Hyperlipidemia is a powerful predictor of CAD with a strong independent, continuous and graded positive association between cholesterol level and risk of coronary events. ${ }^{[3]}$ High Body Mass Index (BMI) has been found to be associated with hypertension. [5] Hypertension is a significant, strong, independent risk factor for CAD. It increases the filtration of lipids from plasma to the intimal cells in presence of hyperlipidemia leading to injury of intima, platelet aggregation and proliferation of smooth muscle cells in the medieval. ${ }^{[6]}$ High salt intake is related to hypertension especially in "salt-sensitive people". Increased sodium / potassium inter relationship is most important predictor of hypertension and coronary ischemia from public health standpoint. ${ }^{[7]}$

The potential association between trace elements status and $\mathrm{CAD}$ has received increased attention. Copper and zinc are involved in cellular utilization of Oxygen, DNA and RNA production, cell membrane integrity and sequestration of free radicals. Magnesium appears to have favorable effects on cardiac arrhythmias coronary blood flow, platelets aggregation and myocardial metabolism. ${ }^{[8]}$ Magnesium was found to be decreased in CAD and Hypertension. ${ }^{[9],[10]}$ Zinc is decreased, while Iron and copper are increased in CAD. ${ }^{[8],[10]}$ Smoking is a powerful risk factor for Cad with risk of events increasing in relation to the number of cigarettes smoked daily. ${ }^{[11]}$ Clustering of the risk factors may increase the risk for Cad more than any of the factors alone. ${ }^{[12]}$

The present work aimed to study the prevalence of some nutritional risk factors as high caloric dietary intake, overweight, hyperlipidemia, hypertension and their correlation with serum concentration of some trace elements as new predictors for coronary ischemia in residents of Jeddah Governorate.

\section{Methodology}

All volunteers of the present study randomly participated from different rural centers (rural group) and from residents of Jeddah town (urban group). They were 880 healthy volunteers (264 rural men, 228 rural women, 156 urban men and 232 urban women). Their ages ranged from $20-60$ years old. During house visits each volunteer was subjected to full medical history taking, history of hypertension, diabetes mellitus, renal diseases or previous cardiac attack. Smoking habits were recorded as, never smoked, current smoker (those who self-reported any smoking in the past month), according to CDC, 2006. ${ }^{\text {[13] }}$

Questionnaire was designed and tested to collect data on diets of the subjects. Actual, recording of the quantity of food and calories consumed by each subject for one complete day was done according to food composition tables. ${ }^{[14]}$ Thorough, clinical examination, body weight, and height measurements were done to all subjects. Body Mass Index (BMI) was calculated as weight in $\mathrm{kg} /$ height in $\mathrm{m}^{2}$. ${ }^{[15]}$ Blood pressure (B.P.) determination was done to each one. Three reads were taken using a standard mercury sphygmomanometer. The average of the last readings defines B.P. using the guidelines established by the fifth report of the Joint National committee (2013) ${ }^{[16]}$ on detection evaluation and treatment of high blood pressure. Subject was considered hypertensive if he had SBP $\geq 140$ $\mathrm{mm} / \mathrm{Hg}$ and / or DBP $\geq 90 \mathrm{~mm} / \mathrm{Hg}$.

Every rural subject, included in the study was instructed to attend the rural health unit of his village. Every urban subject was asked to attend certain hospital in the next day's morning. All subjects are informed to be fasting for about 12 hours. Sample of venous blood was taken and serum was separated and kept frozen at $-20^{\circ} \mathrm{C}$ for subsequent analysis:

Serum total cholesterol, triglycerides, low density lipoprotein cholesterol and high density lipoprotein cholesterol concentrations with an Auto analyzer II (Technicon, Instrument corp., Tarry town, N. Y.).

Serum trace elements (copper, zinc, magnesium, and Iron), are analyzed inductively by Coupled Plasma Mass Spectrometry (ICP-MS). ${ }^{[17]}$

Serum sodium concentration was estimated by flame photometer.

ELIZA estimated serum calcium and phosphorus.

Clustering of risk factors in the studied groups was studied according to National Cholesterol Education Program (2013). ${ }^{[18]}$

Family history of CAD (parent or sibling $\leq 55$ in male and $\leq 65$ in female) age ( $\leq 45$ for male and $\leq 55$ for female).

If the level of HDL-ch was more than $60 \mathrm{mg} / \mathrm{dl}$, subtract one risk factor. No national reference data are available on the numbers of the risk factors.

\section{Results}


Table 1. Analysis of baseline characteristics among the studied participants.

\begin{tabular}{|c|c|c|c|c|c|c|}
\hline & Urban men $N=156$ & Rural men N=264 & $P$ value & Urban women $\mathrm{N}=\mathbf{2 3 2}$ & Rural women $\mathrm{N}=\mathbf{2 2 8}$ & $P$ value \\
\hline \multicolumn{7}{|l|}{ Age: } \\
\hline Range & $21-60$ & $19-60$ & & $18-60$ & $18-60$ & \\
\hline Mean \pm S.D. & $30.7 \pm 18.4$ & $34.94 \pm 19.8$ & & $31.6 \pm 20.7$ & $34.7 \pm 20.5$ & \\
\hline Age $\geq 45$ & $16(10.3 \%)$ & $84(31.8 \%)$ & NS & & & Ns \\
\hline Age $<40$ & $140(89.7 \%)$ & $180(69.2 \%)$ & & & & \\
\hline age $\geq 55$ & & & & $44(19 \%)$ & $84(36.8 \%)$ & \\
\hline Current Smoking & $56(35.89 \%)$ & $128(48.5 \%)$ & 0.05 & Denied & Denied & \\
\hline Non-Smoker & $100(64.1 \%)$ & $136(51.5 \%)$ & NS & $232(100 \%)$ & $228(100 \%)$ & \\
\hline \multicolumn{7}{|l|}{ Family History of CAD: } \\
\hline Negative & $88(55.3 \%)$ & $220(83.3 \%)$ & & $172(74.1 \%)$ & $208(91.2 \%)$ & 0.001 \\
\hline \multicolumn{7}{|l|}{$\begin{array}{l}\text { Family History of Di } \\
\text { abetes Mellitus: }\end{array}$} \\
\hline Positive & $38(24.4 \%)$ & $48(18.2 \%)$ & & $68(29.3 \%)$ & $30(13.2 \%)$ & \\
\hline Negative & $118(75.6 \%)$ & $216(81.8 \%)$ & 0.01 & $164(70.7 \%)$ & $198(86.8 \%)$ & 0.001 \\
\hline \multicolumn{7}{|l|}{ Systolic Blood pressure } \\
\hline Range & $120-160$ & $110-160$ & & $110-170$ & $100-160$ & \\
\hline Mean \pm SD & $138.7 \pm 12.5$ & $142 \pm 21.6$ & NS & $134 \pm 24.3$ & $128 \pm 20.5$ & 0.05 \\
\hline \multicolumn{6}{|l|}{ Diastolic Blood pressure } & 0.01 \\
\hline Range & $70-110$ & $70-110$ & & $60-110$ & $60-110$ & \\
\hline Mean $\pm \mathrm{SD}$ & $95 \pm 18.9$ & $98 \pm 19.4$ & NS & $88 \pm 23.8$ & $78 \pm 22.7$ & 0.01 \\
\hline D. B. p. $>90$ & $30(19.2 \%)$ & $34(12.9 \%)$ & 0.05 & $16(6.9 \%)$ & $36(15.8 \%)$ & 0.01 \\
\hline \multicolumn{7}{|l|}{ BMI: } \\
\hline Range & $18.9-34.2$ & $17.0-28.8$ & & $17.0-46.9$ & $15.9-35.0$ & \\
\hline Mean \pm SD & $24.9 \pm 3.8$ & $22.6 \pm 2.96$ & 0.01 & $27.95 \pm 6.6$ & $22.6 \pm 4.9$ & 0.01 \\
\hline $\mathrm{BMI} \geq 25$ & $64(41.0 \%)$ & $68(25.8 \%)$ & 0.001 & $152(65.5 \%)$ & $64(28.1 \%)$ & 0.001 \\
\hline
\end{tabular}

$\mathrm{NS}=$ Not significant

Table 2. Statistical analysis of daily caloric intake in the studied groups.

\begin{tabular}{|c|c|c|c|c|c|c|c|c|}
\hline \multirow{2}{*}{ Variable } & \multicolumn{2}{|c|}{ Urban men $\mathrm{No}=156$} & \multicolumn{2}{|c|}{ Rural men No=264 } & \multicolumn{2}{|c|}{ Urban women $\mathrm{No}=232$} & \multicolumn{2}{|c|}{ Rural women No $=228$} \\
\hline & Range & Mean & Range & Mean & Range & Mean & Range & Mean \\
\hline Total calories per day & $2114-6037$ & $3850.1 \pm 770.7$ & $1360-5015$ & $3520.73 \pm 752.19$ & $2088-4785$ & $3450.8 \pm 749.4$ & $1980-3930$ & $3025.7 \pm 548.5$ \\
\hline $\mathrm{CHO} \&$ fats calories per day & $926-2954$ & $1675.6 \pm 605.6$ & $890-3292$ & $1986.38 \pm 517.96$ & $588-2954$ & $1557.1 \pm 555.1$ & $600-2616$ & $1631.8 \pm 526.3$ \\
\hline Protein calories per day & $150-1260$ & $350.1 \pm 205.2$ & $135-1027$ & $293.97 \pm 169.34$ & $166-914$ & $306.2 \pm 192.8$ & $135-483$ & $192.5 \pm 97.84$ \\
\hline
\end{tabular}

Table 3. Kidney \& liver function tests for the studied groups.

\begin{tabular}{|c|c|c|c|c|c|c|c|c|}
\hline \multirow{2}{*}{ Parameters } & \multicolumn{2}{|c|}{ Urban Men No. $=156$} & \multicolumn{2}{|c|}{ Rural Men No. = 264} & \multicolumn{2}{|c|}{ Urban Women No $=232$} & \multicolumn{2}{|c|}{ Rural Women No $=238$} \\
\hline & Range & Mean $\pm \mathrm{SD}$ & Range & Mean \pm SD & Range & Mean \pm SD & Range & Mean \pm SD \\
\hline S. Urea (mg/dl) & $20-48$ & $31.05 \pm 6.28$ & $19-58$ & $34.38 \pm 7.58 * *$ & $18-52$ & $34.21 \pm 6.6$ & $19-53$ & $33.19 \pm 7.73$ \\
\hline S. Creatinine. $(\mathrm{mg} / \mathrm{dl})$ & $0.45-1.3$ & $0.71 \pm 0.23$ & $0.4-1.6$ & $0.8 \pm 0.29 *$ & $0.35-1.5$ & $0.78 \pm 0.29$ & $0.35-1.6$ & $0.78 \pm 0.32$ \\
\hline S. AST (IU/L) & $7-19$ & $11.05 \pm 2.78$ & $6.0-29$ & $11.59 \pm 4.57$ & $7.0-20$ & $10.38 \pm 3.26$ & $7.0-26$ & $11.57 \pm 4.18^{*}$ \\
\hline S. ALT (IU/L) & $6-16$ & $12.66 \pm 3.04$ & $4.0-25$ & $13.89 \pm 5.15^{*}$ & $3.0-19$ & $11.9 \pm 2.45$ & $6.0-23$ & $13.9 \pm 4.68 * *$ \\
\hline S. Total bilirubin (mg/dl) & $0.34-0.93$ & $0.64 \pm 0.17$ & $0.32-0.99$ & $0.65 \pm 0.19$ & $0.35-1.0$ & $0.65 \pm 0.16$ & $0.32-0.99$ & $0.68 \pm 0.17$ \\
\hline S. Direct Bilirubin (mg/dl) & $0.1-0.26$ & $0.16 \pm 0.04$ & $0.10-0.26$ & $0.17 \pm 0.04 *$ & $0.1-0.77$ & $0.18 \pm 0.09$ & $0.10-0.29$ & $0.17 \pm 0.05$ \\
\hline S. Alk. Phosph. (IU/L) & 25.96 & $59.03 \pm 10.26$ & $21-95$ & $54.67 \pm 10.01$ & $25-97$ & $58.57 \pm 19.01$ & $24-96$ & $60.78 \pm 21.38$ \\
\hline S. Total Protein (mg/dl) & $6.1-8.4$ & $7.4 \pm 0.6$ & $6.1-8.5$ & $8.5 \pm 0.5$ & $6.2-8.4$ & $7.4 \pm 0.6$ & $6.1-8.4$ & $7.4 \pm 0.7$ \\
\hline S. Albumin (mg/dl) & $2.9-6.0$ & $4.5 \pm 0.9$ & $3.2-5.3$ & $4.5 \pm 0.6$ & $3.4-6.3$ & $4.4 \pm 0.8$ & $3.3-6.3$ & $4.4 \pm 0.8$ \\
\hline
\end{tabular}

N. S. $=$ Not Significant

$* \mathrm{P}<0.05$

$* * \mathrm{P}<0.01$

$* * * \mathrm{P}<0.001$ 
Table 4. Serum trace elements concentration for the studied groups.

\begin{tabular}{lllllllll}
\hline \multirow{2}{*}{ Parameters } & \multicolumn{2}{l}{ Urban Men No. $=\mathbf{1 5 6}$} & \multicolumn{2}{l}{ Rural Men No. $=\mathbf{2 6 4}$} & \multicolumn{2}{l}{ Urban Women No=232 } & \multicolumn{2}{c}{ Rural Women No= 238 } \\
\cline { 2 - 8 } & Range & Mean \pm SD & Range & Mean \pm SD & Range & Mean \pm SD & Range & Mean \pm SD \\
\hline Serum Phosphorus & $27-49$ & $36.29 \pm 6.28$ & $25-50$ & $36.18 \pm 6.89$ & $25-49$ & $36.26 \pm 7.29$ & $24-48$ & $36.36 \pm 7.05$ \\
Serum Iron & $50-148$ & $101.45 \pm 33.5$ & $31-160$ & $101.88 \pm 32.57$ & $51-161$ & $107.59 \pm 33.33$ & $52-161$ & $104.03 \pm 33.42$ \\
Serum Calcium & $8.8-10.1$ & $9.36 \pm 0.43$ & $8.4-10.2$ & $10.4 \pm 0.3$ & $8.8-10.2$ & $10.73 \pm 10.33$ & $8.8-10.2$ & $9.31 \pm 0.44$ \\
Serum Copper & $70-169$ & $104.95 \pm 24.28$ & $70-155$ & $106.3 \pm 23.41$ & $80-152$ & $119.16 \pm 25.35$ & $78-155$ & $111.98 \pm 22.61$ \\
Serum Zinc & $105-159$ & $137.63 \pm 14.06$ & $111-163$ & $139.64 \pm 13.99$ & $114-169$ & $139.50 \pm 14.59$ & $115-166$ & $141.50 \pm 12.65$ \\
Serum Sodium & $135-149$ & $141.5 \pm 7.5$ & $135-150$ & $142.2 \pm 7.9$ & $135-150$ & $142.98 \pm 7.9$ & $135-150$ & $140.98 \pm 7.8$ \\
Serum Magnesium & $17-26$ & $21.7 \pm 2.3$ & $12.5-28$ & $21.4 \pm 4.2$ & $13-26$ & $20.7 \pm 2.1$ & $12-32$ & $18.9 \pm 2.6$ \\
Zinc/ Cupper ratio & $0.8-2.0$ & $1.4 \pm 0.2$ & $0.9-2.33$ & $1.4 \pm 0.9$ & $0.82-2.1$ & $1.22 \pm 0.7$ & $0.83-0.2$ & $1.32 \pm 0.8$ \\
\hline
\end{tabular}

N. S. $=$ Not Significant

$* \mathrm{P}<0.05$

$* * \mathrm{P}<0.01$

$* * * \mathrm{P}<0.001$

Table 5. Serum lipids in the studied groups.

\begin{tabular}{lllllllll}
\hline \multirow{2}{*}{ Parameters } & \multicolumn{2}{l}{ Urban Men No. $=\mathbf{1 5 6}$} & \multicolumn{2}{l}{ Rural Men No. $=\mathbf{2 6 4}$} & \multicolumn{2}{l}{ Urban Women No=232 } & \multicolumn{2}{l}{ Rural Women No= 238 } \\
\cline { 2 - 10 } & Range & Mean \pm SD & Range & Mean \pm SD & Range & Mean \pm SD & Range & Mean \pm SD \\
\hline S. Total Cholesterol (mg/dl) & $166-295$ & $233.0 \pm 26.38$ & $115-293$ & $218.76 \pm 35.31$ & $173-295$ & $235.98 \pm 25.32$ & $177-275$ & $226.98 \pm 24.32$ \\
S. Triglycerides (mg/dl) & $99-171$ & $132.58 \pm 20.76$ & $100-200$ & $127.67 \pm 16.94$ & $102-192$ & $129.48 \pm 17.14$ & $100-250$ & $131.43 \pm 21.58$ \\
HDL-Cholesterol & $24-61$ & $39.95 \pm 9.49$ & $21-61$ & $40.5 \pm 9.62$ & $23-55$ & $38.29 \pm 8.33$ & $28-61$ & $38.67 \pm 5.77$ \\
LDL - cholesterol & $119-190$ & $150.16 \pm 16.06$ & $107-190$ & $148.38 \pm 18.66$ & $111-181$ & $150.26 \pm 16.9$ & $106-181$ & $145.90 \pm 19.6$ \\
T. cholesterol / HDL ratio & $3.9-12.3$ & $6.2 \pm 1.6$ & $1.9-11.2$ & $5.8 \pm 1.5$ & $3.9-11.3$ & $6.5 \pm 0.9$ & $3.8-10$ & $6.0 \pm 0.9$ \\
\hline
\end{tabular}

N. S. $=$ Not Significant

$* \mathrm{P}<0.05$

$* * \mathrm{P}<0.01$

$* * * \mathrm{P}<0.001$

Table 6. Biochemical parameters in Men groups.

\begin{tabular}{|c|c|c|c|c|c|}
\hline \multirow{2}{*}{ Parameters } & \multirow{2}{*}{$\begin{array}{l}\text { Laboratory } \\
\text { range }\end{array}$} & \multicolumn{2}{|l|}{ Low values } & \multicolumn{2}{|l|}{ High values } \\
\hline & & Urban men No $=156$ & Rural men No $=264$ & Urban men No $=156$ & Rural men No $=264$ \\
\hline S. Urea (mg/dl) & $15-45$ & $\mathrm{~N}$ & $\mathrm{~N}$ & $4(2.6 \%)$ & $16(6.1 \%)$ \\
\hline S. Creatinine. (mg/dl) & $0.5-1.5$ & $12(7.7 \%)$ & $36(13.6 \%)$ & $\mathrm{N}$ & $4(1.5 \%)$ \\
\hline S. Total bilirubin (mg/dl) & Up to $1 \mathrm{mg}$ & $\mathrm{N}$ & $\mathrm{N}$ & $\mathrm{N}$ & $\mathrm{N}$ \\
\hline S. Direct Bilirubin (mg/dl) & Upto0.25mgdl & $\mathrm{N}$ & $\mathrm{N}$ & $4(2.6 \%)$ & $4(1.5 \%)$ \\
\hline S. Alk. Phosph. (IU/L) & $21-92$ & $\mathrm{~N}$ & $\mathrm{~N}$ & $16(10.3 \%)$ & $16(6.1 \%)$ \\
\hline S. AST (IU/L) & Up to 12 & $\mathrm{~N}$ & $\mathrm{~N}$ & $44(28.2 \%)$ & $128(48.5 \%)$ \\
\hline S. ALT (IU/L) & Up to 12 & $\mathrm{~N}$ & $\mathrm{~N}$ & $20(12.8 \%)$ & $108(40.9 \%)$ \\
\hline S. Total cholesterol $(\mathrm{mg} / \mathrm{dl})$ & $150-270$ & $\mathrm{~N}$ & $12(4.5 \%)$ & $16(10.3 \%)$ & $16(6.1 \%)$ \\
\hline S. Triglycerides (mg/dl) & $60-165$ & $\mathrm{~N}$ & $\mathrm{~N}$ & $12(7.7 \%)$ & $8(3.03 \%)$ \\
\hline S. LDL (mg/dl) & $108-188$ & $\mathrm{~N}$ & $4(1.5 \%)$ & $4(2.6 \%)$ & $8(3.03 \%)$ \\
\hline S. HDL (mg/dl) & $41-58$ & $80(51.3 \%)$ & $160(60.6 \%)$ & $4(2.6 \%)$ & $8(3.03 \%)$ \\
\hline S.cholesterol / HDL (mg/dl) & & & & $104(66.7 \%)$ & $172(64.2 \%)$ \\
\hline S. Total Protein (mg/dl) & $6.4-8.3$ & $16(10.3 \%)$ & $28(10.6 \%)$ & $8(5.1 \%)$ & $36(13.6 \%)$ \\
\hline S. Albumin (mg/dl) & $3.8-5.4$ & $40(25.6 \%)$ & $32(12.1 \%)$ & $36(23.1 \%)$ & $28(10.6 \%)$ \\
\hline S. Zinc (mic.gm / dl) & $109-167$ & $4(2.6 \%)$ & $\mathrm{N}$ & $4(2.6 \%)$ & $\mathrm{N}$ \\
\hline S. Magnesium (mg / dl) & $16-25.5$ & $\mathrm{~N}$ & $24(9.1 \%)$ & $8(5.1 \%)$ & $28(10.6 \%)$ \\
\hline S. Iron (mic.gm / dl) & $150-168$ & $\mathrm{~N}$ & $16(6.1 \%)$ & $\mathrm{N}$ & $\mathrm{N}$ \\
\hline S. Copper (mic.gm / dl) & $70-140$ & $8(5.1 \%)$ & $20(7.6 \%)$ & $24(15.4 \%)$ & $16(6.1 \%)$ \\
\hline S. Calcium (mic.gm / dl) & $8.8-10.2$ & $88(28.2 \%)$ & $24(9.1 \%)$ & $\mathrm{N}$ & $4(1.5 \%)$ \\
\hline S. Phosphorus (mg / dl) & $25-50$ & $\mathrm{~N}$ & $4(1.5 \%)$ & $\mathrm{N}$ & $8(3.03 \%)$ \\
\hline S. Sodium (meq / L) & $133-155$ & $\mathrm{~N}$ & $\mathrm{~N}$ & $\mathrm{~N}$ & $\mathrm{~N}$ \\
\hline
\end{tabular}

N. S. $=$ Not Significant

$* \mathrm{P}<0.05$

$* * \mathrm{P}<0.01$

$* * * \mathrm{P}<0.001$ 
Table 7. Biochemical parameters in Women groups.

\begin{tabular}{|c|c|c|c|c|c|}
\hline \multirow{2}{*}{ Parameters } & \multirow{2}{*}{$\begin{array}{l}\text { Laboratory } \\
\text { range }\end{array}$} & \multicolumn{2}{|l|}{ Low values } & \multicolumn{2}{|l|}{ High values } \\
\hline & & Urban men No $=156$ & Rural men No $=264$ & Urban men No $=156$ & Rural men No $=264$ \\
\hline S. Urea (mg/dl) & $15-45$ & $\mathrm{~N}$ & $\mathrm{~N}$ & $8(3.4 \%)$ & $24(10.5 \%)$ \\
\hline S. Creatinine. (mg/dl) & $0.5-1.5$ & $20(8.6 \%)$ & $28(12.3 \%)$ & $\mathrm{N}$ & $4(1.8 \%)$ \\
\hline S. Total bilirubin (mg/dl) & $\mathrm{UP}$ to $1 \mathrm{mg} / \mathrm{dl}$ & $\mathrm{N}$ & $\mathrm{N}$ & $\mathrm{N}$ & $\mathrm{N}$ \\
\hline S. Direct Bilirubin $(\mathrm{mg} / \mathrm{dl})$ & Up to0.25 & $\mathrm{N}$ & $\mathrm{N}$ & $8(3.4 \%)$ & $20(8.8 \%)$ \\
\hline S. Alk. Phosph. (IU/L) & $21-92$ & $\mathrm{~N}$ & $\mathrm{~N}$ & $20(8.6 \%)$ & $16(7 \%)$ \\
\hline S. AST (IU/L) & Up to 12 & $\mathrm{~N}$ & $\mathrm{~N}$ & $52(22.4 \%)$ & $112(49.1 \%)$ \\
\hline S. Total cholesterol (mg/dl) & $150-270$ & $\mathrm{~N}$ & $\mathrm{~N}$ & $24(10.3 \%)$ & $12(5.3 \%)$ \\
\hline S. Triglycerides (mg/dl) & $40-140$ & $\mathrm{~N}$ & $\mathrm{~N}$ & $64(27.6 \%)$ & $64(28.1 \%)$ \\
\hline S. LDL $(\mathrm{mg} / \mathrm{dl})$ & $108-188$ & $\mathrm{~N}$ & $4(1.8 \%)$ & $\mathrm{N}$ & $\mathrm{N}$ \\
\hline S. HDL $(\mathrm{mg} / \mathrm{dl})$ & $48-75$ & $192(82.8 \%)$ & $212(93 \%)$ & $\mathrm{N}$ & $\mathrm{N}$ \\
\hline S.cholesterol / HDL(mg/dl) & & & & $172(74.1 \%)$ & $192(48.2 \%)$ \\
\hline S. Total Protein (mg/dl) & $6.5-8.3$ & $12(5.2 \%)$ & $32(14 \%)$ & $16(6.9 \%)$ & $20(8.8 \%)$ \\
\hline S. Albumin (mg/dl) & $3.8-5.4$ & $48(20.7 \%)$ & $56(24.6 \%)$ & $24(10.3 \%)$ & $32(14.04 \%)$ \\
\hline S. Iron (mic.gm / dl) & $150-168$ & $8(3.4 \%)$ & $4(1.8 \%)$ & $\mathrm{N}$ & $\mathrm{N}$ \\
\hline S. Copper (mic.gm / dl) & $80-155$ & $20(8.6 \%)$ & $24(10.5 \%)$ & $4(1.7 \%)$ & $4(1.8 \%)$ \\
\hline S. Calcium (mic.gm / dl) & $8.8-10.2$ & $64(27.6 \%)$ & $44(19.3 \%)$ & $\mathrm{N}$ & $4(1.8 \%)$ \\
\hline S. Phosphorus (mg / dl) & $25-50$ & $20(8.6 \%)$ & $20(8.8 \%)$ & $\mathrm{N}$ & $\mathrm{N}$ \\
\hline S. Sodium (meq / L) & $133-155$ & $\mathrm{~N}$ & $\mathrm{~N}$ & $\mathrm{~N}$ & $\mathrm{~N}$ \\
\hline
\end{tabular}

N. S. = Not Significant

$* \mathrm{P}<0.05$

$* * \mathrm{P}<0.01$

$* * * \mathrm{P}<0.001$

Table 8. Biochemical parameters in Smokers and non-smokers.

\begin{tabular}{|c|c|c|c|c|c|c|}
\hline \multirow{2}{*}{ Parameters } & \multicolumn{2}{|c|}{ Urban men $(n=156)$} & \multirow{2}{*}{ P. value } & \multicolumn{2}{|c|}{ Rural men $(n=264)$} & \multirow{2}{*}{ P. value } \\
\hline & Smokers $(n=56)$ & Non-smokers $(n=100)$ & & Smokers $(n=56)$ & Non-smokers $(n=100)$ & \\
\hline Serum T. bilirubin & $0.59 \pm 0.04$ & $0.66 \pm 0.04$ & $<0.01$ & $0.7 \pm 0.03$ & $0.64 \pm 0.02$ & $<0.05$ \\
\hline Serum. D. bilirubin & $0.15 \pm 0.02$ & $0.16 \pm 0.01$ & NS & $0.16 \pm 0.01$ & $0.18 \pm 0.01$ & NS \\
\hline Serum T. Cholesterol & $235.3 \pm 21.4$ & $232.0 \pm 18.9$ & NS & $234.3 \pm 16.1$ & $224.5 \pm 18.4$ & $<0.05$ \\
\hline Serum Triglycerides & $129.3 \pm 23.5$ & $133.9 \pm 26.7$ & $<0.05$ & $122.2 \pm 14.7$ & $127.5 \pm 18.4$ & $<0.05$ \\
\hline Serum HDL & $37.6 \pm 12.8$ & $41.3 \pm 14.4$ & $<0.05$ & $38.9 \pm 11.8$ & $40.1 \pm 13.3$ & NS \\
\hline Serum LDL & $144.5 \pm 27.6$ & $152.9 \pm 31.0$ & $<0.01$ & $152.3 \pm 20.3$ & $156.1 \pm 22.5$ & NS \\
\hline Serum Sodium & $142.3 \pm 19.8$ & $140.9 \pm 27.9$ & NS & $142.7 \pm 22.6$ & $141.7 \pm 20.4$ & NS \\
\hline Serum Iron & $92.7 \pm 34.3$ & $104.8 \pm 42.1$ & $<0.01$ & $95.5 \pm 28.5$ & $99.8 \pm 34.7$ & $<0.05$ \\
\hline Serum Calcium & $9.3 \pm 1.1$ & $9.4 \pm 1.1$ & NS & $9.3 \pm 0.9$ & $9.3 \pm 1.08$ & NS \\
\hline Serum Copper & $89.8 \pm 44.3$ & $110.4 \pm 48.2$ & $<0.001$ & $108.9 \pm 37.1$ & $97.5 \pm 33.6$ & $<0.05$ \\
\hline Serum Zinc & $144.1 \pm 38.7$ & $134.0 \pm 29.7$ & $<0.001$ & $138.3 \pm 27.7$ & $141.6 \pm 26.9$ & $<0.05$ \\
\hline Serum Magnesium & $22.0 \pm 10.4$ & $21.6 \pm 12.1$ & NS & $22.1 \pm 11.8$ & $20.97 \pm 11.3$ & NS \\
\hline Serum Albumin & $4.7 \pm 1.1$ & $4.5 \pm 1.3$ & NS & $4.7 \pm 1.1$ & $4.4 \pm 1.08$ & NS \\
\hline
\end{tabular}

Table 9. Correlation Coefficient between Body Mass index (BMI) and serum trace elements concentration levels.

\begin{tabular}{|c|c|c|c|c|}
\hline \multirow{2}{*}{ Variable } & \multicolumn{4}{|c|}{ Body Mass index (BMI) } \\
\hline & Urban men $(n=156)$ & Rural men $(n=264)$ & Urban women $(n=232)$ & Rural women $(n=228)$ \\
\hline Serum Zinc (r) & -0.12 & -0.077 & 0.21 & 0.12 \\
\hline $\mathrm{P}$-value & 0.49 & 0.56 & $0.05 *$ & 0.37 \\
\hline Serum Magnesium (r) & -0.02 & 0.11 & 0.22 & -0.14 \\
\hline P-value & 0.89 & 0.39 & $0.05 *$ & 0.29 \\
\hline Serum Iron(r) & -0.08 & -0.06 & 0.08 & 0.01 \\
\hline P-value & 0.64 & 0.63 & 0.55 & 0.96 \\
\hline Serum Copper (r) & 0.30 & 0.01 & 0.03 & -0.13 \\
\hline P-value & $0.05^{*}$ & 0.97 & 0.23 & 0.34 \\
\hline Serum Calcium (r) & 0.14 & 0.12 & 0.09 & 0.014 \\
\hline P-value & 0.42 & 0.34 & 0.50 & 0.73 \\
\hline Serum Phosphorus (r) & -0.29 & -0.01 & -0.19 & 0.21 \\
\hline P-value & $0.05 *$ & 0.94 & 0.16 & 0.12 \\
\hline Serum Sodium (r) & -0.02 & -0.05 & -0.07 & -0.03 \\
\hline P-value & 0.90 & 0.70 & 0.63 & 0.83 \\
\hline
\end{tabular}


Table 10. Correlation Coefficient between daily total Caloric Intake, serum trace elements concentration and Lipid profile data.

\begin{tabular}{lllll}
\hline Variable & \multicolumn{2}{l}{ Daily Total Caloric Intake (K.cal / day) } & & Rural women (n= 228) \\
\cline { 2 - 5 } & Urban men $(\mathbf{n}=\mathbf{1 5 6})$ & Rural men $(\mathbf{n}=\mathbf{2 6 4})$ & Urban women $(\mathbf{n}=\mathbf{2 3 2})$ & 0.03 \\
\hline Serum Zinc $(\mathrm{r})$ & 0.09 & 0.17 & -0.152 & 0.85 \\
P-value & 0.57 & 0.17 & 0.26 & -0.06 \\
Serum Magnesium (r) & -0.035 & 0.17 & 0.17 & 0.67 \\
P-value & 0.84 & 0.19 & 0.19 & -0.09 \\
Serum Iron(r) & -0.13 & -0.25 & -0.07 & 0.48 \\
P-value & 0.45 & $0.05^{*}$ & 0.60 & -0.15 \\
Serum Copper (r) & 0.08 & -0.18 & -0.152 & 0.56 \\
P-value & 0.61 & 0.15 & 0.25 & -0.18 \\
Serum Calcium (r) & 0.36 & 0.13 & 0.06 & 0.19 \\
P-value & $0.03^{*}$ & 0.32 & 0.65 & 0.14 \\
Serum Phosphorus (r) & -0.02 & -0.06 & -0.14 & 0.29 \\
P-value & 0.23 & 0.62 & 0.13 & -0.19 \\
Serum Sodium (r) & -0.22 & 0.02 & -0.02 & 0.16 \\
P-value & 0.19 & 0.93 & 0.88 & 0.13 \\
Serum T. Cholesterol (r) & 0.15 & -0.11 & -0.07 & 0.33 \\
P-value & 0.36 & 0.38 & 0.62 & 0.16 \\
S Triglycerides (r) & 0.13 & -0.33 & -0.07 & 0.22 \\
P-value & 0.42 & $0.007 * * *$ & 0.14 \\
Serum LDL (r) & -0.14 & -0.09 & 0.61 & 0.29 \\
P-value & 0.42 & 0.49 & 0.23 & -0.12 \\
Serum HDL (r) & -0.12 & 0.01 & $0.03 *$ & 0.29 \\
P-value & 0.46 & 0.92 & 0.02 & 0.85 \\
\hline
\end{tabular}

N. S. $=$ Not Significant

$* \mathrm{P}<0.05$

$* * \mathrm{P}<0.01$

$* * * \mathrm{P}<0.001$

Table 11. Prevalence of risk factors in the studied groups.

\begin{tabular}{lllll}
\hline No. of risk factors & Urban men $(\mathbf{n}=\mathbf{1 5 6})$ & Rural men $(\mathbf{n}=\mathbf{2 6 4})$ & Urban women $(\mathbf{n}=\mathbf{2 3 2})$ & Rural women $(\mathbf{n}=\mathbf{2 2 8})$ \\
\hline None & $24(15.4 \%)$ & $48(18.2 \%)$ & $20(8.6 \%)$ & $4.0(1.8 \%)$ \\
One risk factor & $32(20.5 \%)$ & $80(30.3 \%)$ & $24(10.3 \%)$ & $76(33.3 \%)$ \\
Two risk factors & $28(17.95)$ & $36(13.6 \%)$ & $64(27.6 \%)$ & $48(29.8 \%)$ \\
Three risk factors & $32(20.5 \%)$ & $40(15.2 \%)$ & $76(32.8 \%)$ & $36(15.8 \%)$ \\
four risk factors \& more & $40(25.6 \%)$ & $60(22.7 \%)$ & $48(20.7 \%)$ & $64(28.1 \%)$ \\
\hline
\end{tabular}

Risk factors include:

- $\quad$ Age $\geq 45$ in males and $\geq 55$ in females

- $\quad \mathrm{BMI} \geq 25$

- $\quad$ Family history of CAD (parents or siblings)

- $\quad$ Current smoking

- Hypertension

- High levels of one or more parameters of lipids

- Lower levels of HDL-ch $(\leq 35 \mathrm{mg} / \mathrm{dl})$

\section{Discussion}

It remains unknown to what extent traditional risk factors predict CAD in general population in Saudi Arabia, because there have been no long-term prospective studies completed. Few studies offer information on the magnitude of the risk factors problem and the extent to which family members are being effectively treated. The present study reported actual nutritional risk factors values in apparently healthy subjects in Jeddah governorate population. The prevalence of overweight $(\mathrm{BMI} \geq 25)$ in the studied groups was high in urban women $(65.5 \%)$, and urban men (41 \%). The prevalence was less in rural women $(28.1 \%)$, and rural men $(25.8 \%)$. These results were much higher than those of El-Mugamer et al. who found that $27 \%$ of urban population in U. A. E. is obese and the females showed significant higher values of BMI $(\mathrm{P} \geq 0.002) .{ }^{[19]}$ Sood et al. reported that $21.5 \%$ of urban population had BMI $\geq 25$ and obesity correlated significantly with age. ${ }^{[20]}$ In contrast, Ritchie et al. reported that lower values of BMI were found with advanced age due to difficulty in chewing and absence of dentures. ${ }^{[21]}$ The high prevalence of obesity in females appears to be a post-monarchial phenomenon, probably caused by hormonal changes. Socioeconomic status may influence physical activity Patten of subjects. Physical inactivity contributes independently to body fat mass especially in males. ${ }^{[10],[22]}$ There was a graded relationship between male socioeconomic status (defined as educational status) and BMI. While for females, only a low educational level was associated with a higher BMI. ${ }^{[23]}$ Fast food meals and Television viewing hours may be contributing factors for 
obesity especially in women. ${ }^{[24]}$ Energy requirements decline progressively beyond age 50as the lean (muscle) mass declines and resting metabolic energy expenditure decreases. Energy needs for activity also declines as aging often leads to more sedentary life style. ${ }^{[25]}$ Fatness levels at above $25 \%$ in males and $30 \%$ in females are indicative of increased risk for hypertension and increased lipoprotein ratio. ${ }^{[5]}$

In the present study, smoking was more prevalent in rural than urban men $(48.5 \%$ vs. $35.9 \%)$. These results agreed with those of Chadha et al. who recorded high prevalence of passive smoking in rural women with increase risk factor for CAD with aging due to prolonged exposure to tobacco smoking. [26] Meanwhile, Mollentze et al. found that prevalence of current smoking in urban men was almost double that in rural man. ${ }^{[27]}$ Both cigarettes smoking and obesity are adversely affecting other cardiac risk factor. ${ }^{[28]}$ Smoking has been reported to worsen HDL-ch ${ }^{[29]}$, alter trace element metabolism (increase serum $\mathrm{Cu}^{+2} \& \mathrm{Zn}^{+2}$ ) as well as components of free radicals defense system. ${ }^{[11]}$

In the present study, the prevalence of systolic hypertension $(\geq 140 \mathrm{~mm} / \mathrm{Hg}$ ) was significantly higher in urban $(30.8 \%)$, than in rural men $(25.8 \%)$ and was significantly less prevalent in urban $(13.8 \%)$ than in rural women $(24.6 \%)$. Diastolic hypertension $(\geq 90 \mathrm{~mm} / \mathrm{Hg})$ was insignificantly more prevalent in urban $(19.2 \%)$ than in rural men $(12.9 \%)$ and was less prevalent in urban $(6.9 \%)$ than in rural women $(15.8 \%)$. These results agreed with Khalifa, a study that was done in rural area in El-Mania Governorate, Upper Egypt, who found that systolic hypertension was present in $20.3 \%$ of rural men and $13.4 \%$ of rural women. Also, he found that diastolic hypertension was found in $10.2 \%$ of rural men and $12.4 \%$ of rural women. ${ }^{[30]}$ But our results were higher than those previously recorded by Rizk et al. that systolic hypertension was in $23.7 \%$ and diastolic hypertension was in $18.3 \%$ of general population. ${ }^{[31]}$

Family history of CAD was less recorded in rural subjects ( $16.7 \%$ of rural men and $8.8 \%$ of rural women). This may be attributed missing cases due to fault in diagnosis, and deficient coronary care units. Again, family history of diabetes mellitus was more prevalent in urban subjects: women more than in men $(29.3 \%$ vs. $24.4 \%)$. These results were higher than that mentioned by Khalifa who reported that the diabetes mellitus was $5.5 \%$ in rural women and was 3.4 in rural men. ${ }^{[30]}$ Serum bilirubin level was found to be within normal laboratory range in all studied groups. Only direct bilirubin was significantly higher in rural than in urban men $(0.17 \pm 0.04$ vs. $0.16 \pm 0.04)$. A mild increase in serum bilirubin has been suggested as a protective factor, possibly reducing the risk of $\mathrm{CAD}$, by acting as an antioxidant. This protective effect is comparable to that of HDL-ch. Moreover, cigarette smoking decreases serum bilirubin, hence decreases its protective effect. ${ }^{[32]}$ In the present study, smokers showed significant low of serum total bilirubin than non-smokers $(0.59 \pm 0.04$ vs. $0.66 \pm 0.04)$. Such finding was not found in rural smokers (table, 8 ).

Lowered albumin concentration was found in $25.6 \%$ of urban men, $12.1 \%$ or rural men, $20.7 \%$ of urban women and
$24.6 \%$ of rural women. A similar result was found before in studies of Ritchie et al., who recorded that $19.5 \%$ of normal population had low serum albumin concentration. ${ }^{[21]}$ There was a highly significant inverse relationship between serum albumin level and risk of CAD. Individual with a baseline level of serum albumin $\geq 4.7 \mathrm{mg} / \mathrm{dl}$ has an odd ratio of 0.45 as compared with individual with a baseline level of serum albumin less than $4.4 \mathrm{mg} / \mathrm{dl}$. The relation persisted after adjusting for other risk factors (e.g. smoking, blood pressure, and serum cholesterol level). ${ }^{[33],[34]}$ In the present study, no relation was found between serum total protein, serum albumin and smoking. Lower albumin levels maybe a marker of persistent injury to arteries and progression of atherosclerosis as it is related to acute phase reaction. ${ }^{[33]}$ There was a positive significant correlation between hypercholesterolemia and reduction of the transport function of serum albumin. ${ }^{[35]}$ Hypercreatininemia was observed only in $1.5 \%$ of rural men and 1.85 of rural women. It is considered an independent risk factor for CAD. [7],[36] Hypercreatininemia, more than $1.7 \mathrm{mg} / \mathrm{dl}$ in hypertensive people may be a stronger CAD factor than diabetes, smoking, left ventricular hypertrophy $\mathrm{r}$ systolic blood pressure. ${ }^{[7]}$ Each $0.1 \mathrm{mg}$ dl $(9 \mathrm{mmol} / \mathrm{L})$ increment in the baseline serum creatinine, increase the relative risk for subsequent $\mathrm{CAD}$ mortality by $47 \%$. Moreover, serum creatinine value obtained in normotensive, non- obese normoglycemic, survivors of myocardial infarction without preexistent renal disease or heart failure, provides independent prognostic information regarding subsequent CAD mortality. ${ }^{[36]}$

Serum total cholesterol was higher than the normal laboratory range $1.3 \%$ of urban men \& women, $6.1 \%$ of rural men and $5.3 \%$ of rural women. Higher level of serum triglycerides was prevalent in rural women $(28.1 \%)$ and urban women $(27.6 \%)$, but was less prevalent in both men groups $(7.7 \%$ and $3.03 \%)$. There is no any raise in levels of serum LDL-ch in women groups, but it only raised levels were seen in $3.5 \%$ of rural men and $2.6 \%$ of urban men. Lowered concentrations of LDL-ch were much prevalent in rural women (93\%), and urban women (82.8 5), than obviously reported by Chadha et al. ${ }^{[26]}$ They attributed their results as rural subjects had higher total caloric and saturated fats intakes than urban subjects. Meanwhile, the present results disagreed with those of Chuang et al., who found that increased LDK-ch and decreased HDL-ch were more prevalent in urban than rural population. ${ }^{[37]}$ Also, disagreed with those of Singh et al., who found that HDL-ch was raised in urban than in rural population. They explained their results by the better socioeconomic status of urban population; the latter consume higher total and saturated fats, refined carbohydrate and lower total and complex carbohydrate, compared to rural population. ${ }^{[10]}$ The total caloric intake per day showed a high significant correlation with serum triglycerides in rural men group only, but insignificant correlation with other parameters of lipid profile. These results were agreed with those of Petridou et al., who concluded that there is no clear evidence of effect of qualitative aspects of diet and total cholesterol LDL-ch and 
HDL-ch. ${ }^{[4]}$

Serum cholesterol / HDL ratio was more than $5 \%$ and it is prevalent in rural and urban women $(84.2 \%$ and 74.1 respectively). It was also less prevalent in rural and urban men $(64.2 \%$ and $68.4 \%$ respectively). Prevalence of CAD and extra coronary atherosclerosis increases in those with ratio more than $5 \%$. Urban smokers showed significant low serum levels of HDL-ch $(37.6 \pm 12.8$ vs. $41.3 \pm 14.4)$, than non-smokers. Meanwhile, rural smokers showed significant raised serum levels of total cholesterol $(234.3 \pm 18.4$ vs. $224.5 \pm 18.4)$. High serum total cholesterol, triglycerides with low levels of HDL-ch are considered high risk factors for CAD. ${ }^{[25],[26],[38]}$

The role of trace elements in predicting CAD has been extensively debated. High serum $\mathrm{Cu}^{+2}$ and $\mathrm{Fe}^{+2}$ with low serum $\mathrm{Zn}^{+2}$ and $\mathrm{Mg}^{+2}$ are associated with CAD, while serum $\mathrm{Ca}^{+2}$ and $\mathrm{ph}^{+2}$ have no such correlation. ${ }^{[10],[39]}$ Recently, Mahalingam et al. concluded that serum $\mathrm{Mg}^{+2}$ and $\mathrm{Na}^{+2}$ are decreased in high risk subjects with CAD. ${ }^{[40]}$ Iron and $\mathrm{Zn}^{+2}$ are necessary for oxidized LDL-ch and lipid peroxides, hence raised levels in hyperlipidemia carry risk for occurrence of CAD. ${ }^{[41]} \mathrm{Cu}^{+2}$ and $\mathrm{Zn}^{+2}$ tend to increase in serum of patients with $\mathrm{CAD}$ as they are shifted from peripheral atherosclerotic lesions. ${ }^{[42]}$ In the present study, serum $\mathrm{Zn}^{+2}, \mathrm{Ph}^{+2}$ and $\mathrm{Fe}^{+2}$ showed insignificant differences between the studied group, meanwhile, serum $\mathrm{Ca}^{+2}$ showed significant rise in rural that urban men(10.4 \pm 0.3 vs. $9.4 \pm 0.4)(\mathrm{P} \geq 0.05)$. Also, serum $\mathrm{Cu}^{+2}$ was significantly raised in urban than rural women $(119.2 \pm 25.4$ vs. $111.98 \pm 22.6)(\mathrm{P} \geq 0.05)$ respectively. These results disagree with that of Singh et al., who found a significant lower $\mathrm{Zn}{ }^{+2}$, and higher $\mathrm{Cu}^{+2}$ and $\mathrm{Fe}^{+2}$ serum concentrations, in rural population. ${ }^{[10]}$ As regards serum $\mathrm{Mg}^{+2}$, it was significantly raised in urban than rural women $(20.7 \pm 2.1$ vs. $18.9 \pm 2.6)(\mathrm{P} \geq 0.05)$. This result agrees with that of Singh et al. ${ }^{[10]} \mathrm{Zn}^{+2}$ and $\mathrm{Cu}^{+2}$ ratios showed insignificant differences between the studied groups $(1.4 \pm$ 0.2 vs. $1.4 \pm 0.9$ for men and $1.2 \pm 0.7$ vs. $1.3 \pm 0.8$ for women). But in Singh et al. such ratio was significantly less in rural than urban population $(0.58 \pm 0.08$ vs. $1.1 \pm 0.25) .{ }^{[10]}$ Serum $\mathrm{cu}^{+2}$ was lower than normal laboratory range in $7.6 \%$ of rural men, $5.1 \%$ of urban men, $10.5 \%$ of rural women and $8.6 \%$ of urban women. Serum $\mathrm{Zn}^{+2}$ were within normal laboratory range in all studied groups. SerumCu ${ }^{+2}$ and $\mathrm{Zn}+{ }^{2}$ concentrations are normally constant throughout life. They decreased only in malnutrition, catabolic states and malabsorption syndromes. ${ }^{[43]}$ In the present study, serum $\mathrm{Cu}^{+2}$ decreased and serum $\mathrm{Zn}^{+2}$ increased in urban smokers, while the reverse is a true rural smoker (table: 8). these results disagreed with those of Dubick and Keen, who found that serumCu $\mathrm{Cu}^{+2}$ and $\mathrm{Zn}^{+2}$ were significantly raised in smokers than non-smokers. ${ }^{[1]}$

In the present study, BMI showed significant correlation with serum $\mathrm{Cu}^{+2} \& \mathrm{Ph}^{+2}$ in urban men and $\mathrm{Zn}^{+2} \& \mathrm{Mg}^{+2}$ in urban women $(\mathrm{P} \geq 0.05)$. This result agreed with that of El-Carte-Lopez et al., who found such a significant correlation $(\mathrm{P} \geq 0.01)$ with $\mathrm{Cu}^{+2} \& \mathrm{Zn}^{+2}$ that was markedly amplified if adiposity parameters are taken into account. ${ }^{[44]}$
Total caloric intake per day correlated significantly with serum $\mathrm{Ca}^{+2}(\mathrm{P} \geq 0.03)$, in urban men, and inversely correlated with serum $\mathrm{Fe}^{+2}(\mathrm{P} \geq 0.05)$ and serum TG $(\mathrm{P} \geq 0.007)$ in rural men.

The relation between diet, trace elements and CAD is complex. The intake of saturated fats is associated with increase myocardialCu ${ }^{+2}$ concentrations. ${ }^{[39]}$ Meanwhile, dietary $\mathrm{Mg}^{+2}$ may have contributed to the reduction of serum total cholesterol, LDL-ch, TG, and marginal rise in HDL-ch. ${ }^{[10]}$ High iron nutritional status, with low $\mathrm{Cu}^{+2}$ nutritional status may predispose to $\mathrm{CAD}$, as iron acts as proxidants, while helps to maintain oxidant / antioxidant balance. ${ }^{[45]} \mathrm{On}$ the other hand, Sempos et al. and Rheunanen et al. recorded that; there is no association between excess iron intake, excess body iron stores and CAD. ${ }^{[46],[47]}$ Their findings supported previous findings of NHANES study that afforded the hypothesis of a positive iron-CAD relationship. ${ }^{[42]}$

The high prevalence of four or more risk factors was found in rural women $(28.1 \%)$ and urban men $(25.6 \%)$, while urban women showed high prevalence of three risk factors. The prevalence of major risk factors increased in rural areas in the beginning of the last decade due to dietary and life-style changes that have taken place in the last thirty years. [48]

\section{Recommendations}

Primary prevention is aimed at smoking cessation, diet modification and increase physical activity. ${ }^{[49]}$ Control of the major risk factors mainly in those with clustered factors will substantially reduce the risk of ischemic events. ${ }^{[1]}$ Screening with total levels is most likely to be useful when done in middle aged people with multiple risk factors. In these populations, cholesterol reduction appears to be effective and cost-effective. ${ }^{[50]}$ The new dietary advice should include: reduce intake of total (not more than $30 \%$ of energy) and saturated (less than 10\%) fats, maintain minimal intake of essential Omega-6 fatty acids, increase consumption of Omega-3 fatty acids, augment intake of natural oxidants sufficient intake of vegetable and plant proteins. [51] Mono-unsaturated oils such as olive oil or canola oil are desirable as they do not increase the risk of atherosclerosis. [52]

\section{References}

[1] Gensini G. F., Comeglio M. and Colella A. (2008): Eur.Heart J. suppl. 19: 53 .

[2] Green, M. S. (2008): Public health Rev., 26 (1): 31

[3] Sharpnel W. S., Calvert G. D.,Nestel, P. J. and Truswell, A. S.(2012): Med. J. Aust. 156 (suppl.) 9.

[4] Patridou, E., Malamou H., Doxiadis S. and Flytzani V., (2005): Ann. Epidemiol. 5 (4): 286.

[5] Gunnel D. J., Frankel S. J., Peter T. J. and Nanchahal K. (2008): Am. J. Clin.nutr. 67 (6): 111 
[6] Cheitlin M. D., Sokolow M. and Mcllrov M. B. (2013): Coronary heart diseases. Un "Clinical Cardiology" 6th Ed. Prentice Hall Intermat, Inc.:147

[7] Hall W. d. (2009): Am. J. Med. Sci. 317 (3):176

[8] He B. d., Zhao D. S. and Zhao L. (2004): Chung Hua 1Hsueh Tsa Chih 74 (8): 492

[9] Saito N. (2006): Nippon rinsho Jan. 54 (1): 59

[10] Singh R. B. Gupta U. c., Mittal N. and Niaz M. A. (2007): J. Am. Coll. Nutr. 16: 162

[11] Dubick M. a. and Keen C. L. (2011): Biol. Trace Element res. 31 (2): 97

[12] Jousilahti p., Vartiainen E., Tuomilefto J. and Piska P. (2005): J. Cardiovasc. Risk, 2 (1): 63

[13] CDC (2006): Behavioral risk factor surveillance selected states. MMWR. 235: 253

[14] Food and Nutrition Board (1989): National Research council Recommended Dietary Allowances. 10th Ed. Washington DC. National Academy Press.

[15] Garrow J. S. and Webester j. (2005): Int. J. Obes. 9 (2): 147

[16] Joint National Committee (JNC) (2013): Arch. Intern. Med.153: 154

[17] Szabo G., Balint S., Nyeste E. and Medgyesi I. (2011): Orv. Hetil. 132 (8): 395

[18] National Cholesterol education Program (2013): Second Report for the expert panel on detection, evalution and treatment of high blood cholestetrol in adults (adult treatment panel II). National Institute of health, heart, lung and blood. JAMA 269: 3015 - 3023 .

[19] El-Mugamer I. T., Ali-Zayat A. S., Hssain M. M. and Pugf R. H. J. (2005): Tro.Med. Hyg. Dec.98 (6): 407

[20] Sood R. K., Gupta A. k., Ahluwalia S. K. and Sood K. C. (2008): Published Erratum Appears in Indian J. Med. Sci. 5 (12) 341.

[21] Ritchi C. S., Burgio K. L., Locher J. L. and Cornwell L. (2007): Am. J. Clin. Nutr. 66 (4): 815.

[22] Al-Nuaim A. R. (2007): Diabt. Med. Jul. 14 (7): 211.

[23] Sundquist J. and Johnsson S. E. (2008): Int. J. Epidemiol 27 (1): 57.

[24] Jeffry R. W. and Frenchn S. A. (2008): Am. J. Public Health. 88 (2): 277.

[25] Fletcher A. E. and Bulpitt C. J. (2012): J. Hypertens Suppl. April (10): 551.

[26] Chadha S. L., Gopinath N. and Shekhawat S. (2007): Bull. World Health Organ. 75 (1): 31.

[27] Mollentze W. F., Moore A. J. and Styn A. F. (2005): S. Africa Med. J. 85 (2): 90.

[28] Menotti A. and Giampaoli S. G. (2008): Ital. Cardiol. 28 (12): 1354.
[29] Willems J. P., Hunt D. E. and Schorling J. B. (2007): Natl. Med. Assoc. 89 (1): 37.

[30] Khalifa M. A. (2005): El-Mania Med. Bull. 6 (1): 97.

[31] Rizk H., Ibrahim M. M., Helmy S. and Kandil H. (2005): How common is hypertension in Egypt? Preliminary results from pilot studies of the Egyptian National Hypertension Project (NHP). E. H. S. 1 (1): 1 - 7.

[32] Hopkins P. N., Wu L. L., Hunt S. C. and James B. C. (2006): Throm. Vasc. Bio. 1 (2): 250.

[33] Kuller L. H., Eichner J. E., Orchard T. J. and Grandits G. A. (2011): Am. J. Epidemiology. 134 (11): 1266.

[34] Corti M. C., Saive M. E. and Guralink J. M. (2006): J. clinical Epidemiology. 49 (5): 519.

[35] Pudiak E. A., Serdiuk N. Z. and Bakaliuk O. I. (2010): Vrach Delo 7: 24.

[36] Matts J. P., Karnegis J. N., Campos C. T. and Fitch L. L. (2013): J. Fam. Pract. 36 (5): 497.

[37] Chuang C. Z., Subramaniam P. N., LeGardeur B. Y. and Lopez A. (2010): Indian Heart J. 500 (3): 285.

[38] Baggio G., Donazzan S., Monti D., Mari D. and Martini S. (2008): Faseb J. Apr. 12 (6): 433.

[39] Alfathan G., Pesonin E., Neuvonen P. J. and Hirvonen J. (2012): J. Trace Elements Electrolytes Health dis. Mars 6 (1): 45.

[40] Mahalingam T. R., Vijayalakshmi S., Prabhu R. K. and Thiruvengadasami A. (2007): Biol. Trace elem. Res. 57 (3): 207.

[41] Miyake N. (2006): Nippon Rinsho. 54 (2): 73.

[42] Liao Y., Cooper R. S. and McGee D. L. (2004): Am. J. Epidemiology. 139 (7): 704.

[43] Falchuk K. H. (2004): Disturbances in trace elements metabolism. In "Harrison's Principlees of Internal Medicine" 13 th Ed. McGrow-Hill Inc. N. Y. London. Page: 481.

[44] El-Carte Lopez T., Villa E. I. and Gost-Grade J. I. (2007): Acta Pediatr. 86 (3): 248.

[45] Strain J. J. (2004): Br. J. Biomen. Sci. 51 (3): 241.

[46] Sempos C. T., Looker A. C., Gillum R. F. and Makuc D. M. (2004): N. Engl. Med. 330 (16): 1119.

[47] Reunanen A., Takkunen H. and Knekt P. (2005): J. Intern. Med. 238 (3): 223.

[48] Voukiklaris G. E., Kafatos A. and Dontas A. S. (2006): Angiology 47: 443.

[49] Howes D. G. (2008): Lippincotts Prim, Pract 2 (5): 514.

[50] Garber A. M., Browner W. S. and Hully S. B. (2006): Ann. Intem. Med. 124 (5): 518.

[51] De-Logeril M. Salen P., Monjaud I. and Delaye J. (2007): Eur. Heart j. 18 (1): 13.

[52] Weisburger J. H (2008): Proc. Soc. Exp. Biol. Med. 218 (2): 140. 